\title{
Differences in first neighbor orientation behind the anomalies in the low and high density trans-1,2-dichloroethene liquid
}

Cite as: J. Chem. Phys. 136, 124514 (2012); https://doi.org/10.1063/1.3697849

Submitted: 30 January 2012 . Accepted: 09 March 2012 . Published Online: 30 March 2012

M. Rovira-Esteva, N. Arul Murugan, L. C. Pardo, S. Busch, J. Ll. Tamarit, G. J. Cuello, and F. J. Bermejo

\section{Lock-in Amplifiers up to $600 \mathrm{MHz}$}
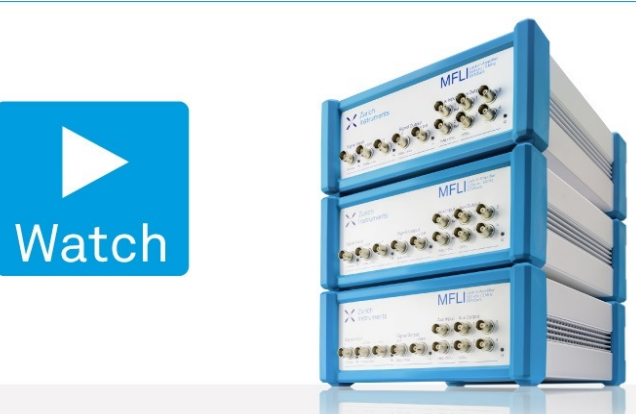


\title{
Differences in first neighbor orientation behind the anomalies in the low and high density trans-1,2-dichloroethene liquid
}

\author{
M. Rovira-Esteva, ${ }^{1}$ N. Arul Murugan, ${ }^{2}$ L. C. Pardo, ${ }^{1}$ S. Busch, ${ }^{3}$ J. LI. Tamarit, ${ }^{1}$ \\ G. J. Cuello, ${ }^{4,5}$ and F. J. Bermejo $0^{4,6}$ \\ ${ }^{1}$ Grup de Caracterització de Materials, Departament de Física i Enginyeria Nuclear, ETSEIB, \\ Universitat Politècnica de Catalunya, Diagonal 647, E-08028 Barcelona, Catalonia, Spain \\ ${ }^{2}$ Division of Theoretical Chemistry and Biology, School of Biotechnology, Royal Institute of Technology, \\ S-106 91 Stockholm, Sweden \\ ${ }^{3}$ Physik Department E13 and Forschungs-Neutronenquelle Heinz Maier-Leibnitz (FRM II), \\ Technische Universität München, Lichtenbergstr. 1, D-85748 Garching, Germany \\ ${ }^{4}$ Facultad de Ciencia y Tecnología, Universidad del País Vasco/EHU, P. Box 644, E-48080 Bilbao, \\ Basque Country, Spain \\ ${ }^{5}$ Institut Laue Langevin, 6 Rue Jules Horowitz, BP. 156, F-38042 Grenoble Cedex 9, France \\ ${ }^{6}$ Instituto de Estructura de la Materia, C.S.I.C., Serrano 123, E-28006 Madrid, Community of Madrid, Spain
}

(Received 30 January 2012; accepted 9 March 2012; published online 30 March 2012)

\begin{abstract}
Trans-1,2-dichloroethene $(\mathrm{HClC}=\mathrm{CClH})$ has several structural and dynamic anomalies between its low- and high-density liquid, previously found through neutron scattering experiments. To explain the microscopic origin of the differences found in those experiments, a series of molecular dynamics simulations were performed. The analysis of molecular short-range order shows that the number of molecules in the first neighbor shell is 12 for the high-density liquid and 11 for the low-density one. It also shows that the angular position of the center of mass of the first neighbor is roughly the same although the molecular orientation is not. In both liquids the first neighbor and its reference molecule arrange mainly in two configurations, each being the most probable in one of the liquids. First neighbors in the configuration that predominates in the high-density liquid tend to locate themselves closer to the reference molecule, an evidence that they are more strongly bonded. This arrangement facilitates a better packing of the rest of molecules in the first neighbor shell so that on average an additional molecule can be included, and is proposed to be the key in the explanation of all the observed anomalies in the characteristics of both liquids. (C) 2012 American Institute of Physics. [http://dx.doi.org/10.1063/1.3697849]
\end{abstract}

\section{INTRODUCTION}

Description of short-range order in molecular liquids poses a highly challenging conundrum. The need to determine not only the correlation between the molecular centers of mass but also the relative molecular orientations to provide a complete picture of the structure of the liquid makes that, even when considering reasonably simple molecules, the number of degrees of freedom quickly increases with respect to mono- or polyatomic liquids. These orientational correlations are usually strongly dependent on the distance for the closest neighbors but have no correlation for the farther ones.

The molecular arrangement has in turn a vast impingement on the dynamics of a system. ${ }^{1,2}$ Thus, a detailed and careful analysis of molecular short-range order is essential to the proper understanding of any system. Several different approaches are used to study molecular short-range order, such as reverse Monte Carlo, ${ }^{3,4}$ empirical potential structure refinement, ${ }^{5,6}$ and molecular dynamic simulations. ${ }^{7,8}$

Trans-1,2-dichloroethene (TDCE) $(\mathrm{HClC}=\mathrm{CClH})$ is a molecular liquid which manifests several anomalies in the behavior of its high-density liquid when compared with its low- density liquid. The molecular geometry of TDCE obtained from an $a b$ initio calculation in the gas phase can be seen in the inset of Fig. 1, where calculated intramolecular angles and distances are shown. First claims of an anomalous behavior in liquid TDCE between 243 and $257 \mathrm{~K}$ were made based on a small density singularity, and a local minimum of the spinlattice relaxation time $\left(T_{1}\right)$ measured by NMR, as well as a $T_{1}$ discontinuity, which was assigned to the appearance of a nematic-like molecular ordering where the molecules have no rotational freedom..$^{9,10}$ A later measurement found a change in the slope of the density but no discontinuity, although the study did find a discontinuity in some dynamic magnitudes as viscosity or as the slope of the rotational relaxation time measured by Raman spectroscopy.

It was therefore concluded that dynamic planar structures were likely to form. ${ }^{11}$ An infrared vibrational spectroscopy study also accounted for a clear discontinuity in several bands, attributed to the existence of a weak $\mathrm{Cl}-\mathrm{H}$ hydrogen bond which induces the formation of dimers with a dipolar moment giving rise to local ordering. ${ }^{12}$ These locally favored structures increase the molecular cooperativity, and thus their reduced mass, slowing down the molecular motion. Kawanishi et al. had even suggested the existence of a liquid-liquid transition, ${ }^{9,10}$ but a recent optical Kerr-effect experiment 


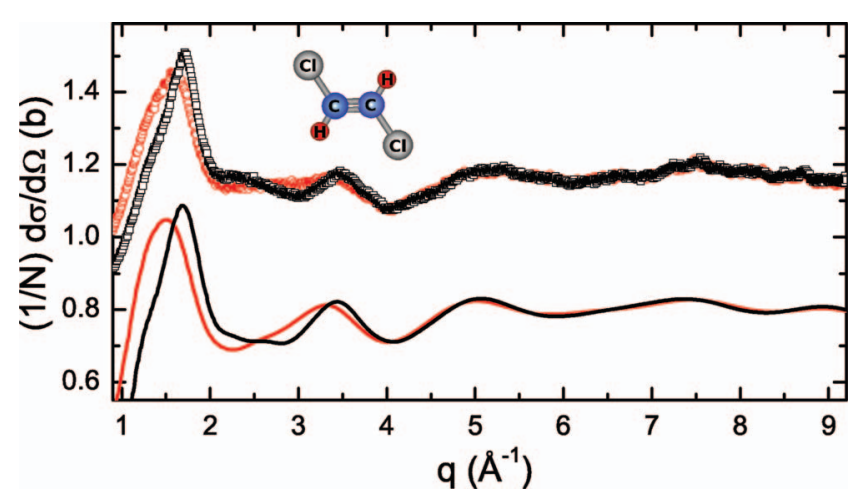

FIG. 1. Differential cross section per atom obtained at the D20 diffractometer (points, with a translation of $+0.4 \mathrm{~b}$ ) and from molecular dynamics (lines) for the low- (red) and high- (black) density liquid. The inset shows an $a b$ initio calculation of the molecular structure of TDCE in the gas phase. Close reproduction of the features in the experimental differential cross section per atom validates the results obtained from analysis of the simulation.

reported that, although it is not a simple liquid, there is no sign of a first order transition in TDCE. ${ }^{13}$

Some of the authors of the present work reported calorimetric and nonlinear dielectric results (a maximum on $c_{p}$ and a strong pre-transitional anomaly, respectively), and suggested that multimolecular heterogeneities (fluctuations with dielectric permittivity slightly different from the "isotropic" surrounding) appear in the high-density TDCE liquid. ${ }^{14}$

Structure and dynamics of TDCE molecules in the highand low-density liquids have been approached through neutron scattering measurements and molecular dynamics simulations in a previous work. ${ }^{15}$ There, diffraction experiments yielded structural changes in the molecular short-range order domain, although no molecular clustering was observed. Experiments on the dynamics showed that the low-density liquid could be well described through a diffusion motion plus an isotropic rotation, whereas in the high-density liquid a confined motion due to cooperative molecular behavior had to be added. Analysis of molecular dynamics simulations showed that molecules are much more ordered in the high-density liquid, and revealed the appearance of two minima in its velocity autocorrelation function, which is characteristic of a backscattering effect. Since the density increase is too small to account for such backscattering effect, this result supports that the confined motion found in the high-density liquid can only be accounted for through a change in the short-range order.

Although it was rather clear that the dynamic anomalies found between the high- and low-density TDCE liquids come down to a change in the molecular arrangement, the precise nature of this structural difference was not analysed. The aim of the present study is to focus on the microscopic structure and elucidate the details behind these anomalies in the high- and low-density TDCE liquids. Neutron diffraction experiments of liquid TDCE performed in both density domains, together with molecular dynamics simulations closely reproducing the experiments, have allowed a thorough statistical analysis of the simulation configurations, unraveling the structural differences in the molecular short-range order of the liquid at both densities.

\section{EXPERIMENT AND SIMULATION DETAILS}

Neutron diffraction experiments on the short-range order of the low- and high-density TDCE liquid were carried out with consistent results at the diffractometer dedicated to glasses and liquids D4c (Ref. 16) and at the high-intensity two-axes diffractometer D20, ${ }^{17}$ both at the Institute LaueLangevin (ILL, Grenoble, France). A $99.70 \%$ purity sample was purchased from Acros Organics (Geel, Belgium) and deuterated to $99 \%$ for both experiments.

Details of the measurement performed at the D4c diffractometer and the obtained results have been published in Ref.15. At D20, an incident neutron wavelength of $\lambda$ $=1.30 \AA$ was used together with a radially oscillating collimator. In order to correct and normalize the data, an empty sample holder and a vanadium rod were also measured. Absorption and multiple scattering corrections and the normalization of the data were performed using the program CORRECT. ${ }^{18}$

A series of molecular dynamics simulations of the lowand high-density TDCE liquid were performed using the program AMBER 8 (Refs. 19, 20) with a simulation box containing 3629 molecules and a time step of 1 fs for the integration of the equations of motion. The inter- and intramolecular interactions for the TDCE molecule were described using a general AMBER force field. ${ }^{19,20}$ The simulations were carried out for the temperature range $200-350 \mathrm{~K}$ in the $N P T$ ensemble, therefore allowing the box size to change, and the total time of each simulation run was $40-50 \mathrm{~ns}^{21,22}$ This simulation is the same as that used in Ref. 15 for the preliminary analysis of the short-range order in liquid TDCE.

The differential cross sections per atom obtained from the simulations for the low- and high-density liquids are shown in Fig. 1 together with the experimental ones, so they can be compared. The structural differences between the low- and high-density liquids in the short-range order domain, already established in a previous work, ${ }^{15}$ can be observed. As can also be seen in this figure, agreement of the simulation with the experimental data is extremely good and absolute intensities are the same within the error. This close agreement provides a solid ground for a detailed analysis of the simulation in search of a microscopic explanation.

\section{ANGLE DEFINITIONS}

To study the short-range order of molecules in the lowand high-density TDCE liquids, two kinds of degrees of freedom have been analysed separately: those regarding the relative positions of the molecules and those regarding their relative orientations.

All the angles used in the analysis of the relative positions and orientations of the molecules obtained from the simulation have been depicted in Fig. 2.

The angles describing the relative position of the center of mass of a molecule with respect to the reference molecule, $\theta_{\mathrm{CM}}$ and $\varphi_{\mathrm{CM}}$, can be seen in Fig. 2(a). $\theta_{\mathrm{CM}}$ is the angle between the axis defined by the chlorine atoms of the reference molecule and the vector that goes through the centers of mass of both molecules. $\varphi_{\mathrm{CM}}$ is the angle between the plane that 


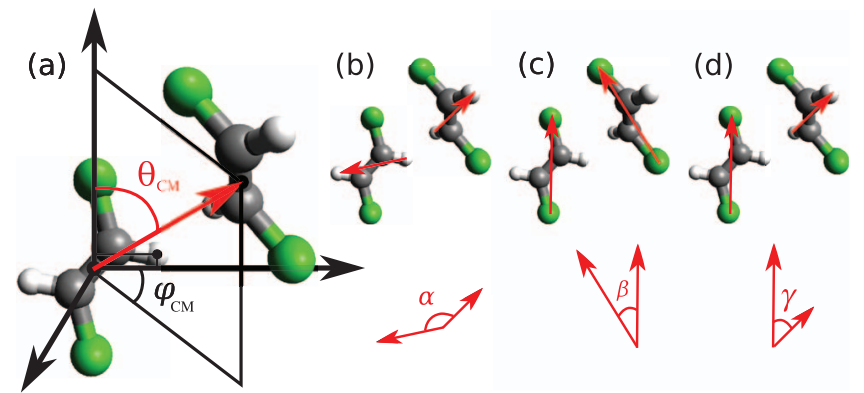

FIG. 2. (a) Angles $\theta_{\mathrm{CM}}$ and $\varphi_{\mathrm{CM}}$ describing the position of the center of mass of one molecule with respect to the center of mass of the reference molecule. (b) Angle $\alpha$ between the axes formed by the hydrogen atoms of both molecules. (c) Angle $\beta$ between the axes formed by the chlorine atoms of both molecules. (d) Angle $\gamma$ between the axes formed by the hydrogen atoms of a molecule and the chlorine atoms of the reference molecule.

contains the whole reference molecule and the plane that contains the chlorine-chlorine axis of the reference molecule and the center of mass of the other molecule. $\theta_{\mathrm{CM}}$ and $\varphi_{\mathrm{CM}}$ will be referred to as angular position of the centers of mass to stress the fact that the relative distance between the molecular centers of mass is already fixed when the neighbors are analysed individually.

The angles $\alpha, \beta$, and $\gamma$ describing the relative orientation between both molecules have been depicted in Figs. 2(b)$2(\mathrm{~d})$, respectively. $\alpha$ is the angle between the axes defined by the hydrogen atoms of each molecule, $\beta$ is the angle between the axes defined by the chlorine atoms of each molecule, and $\gamma$ is the angle between the axis defined by the chlorine atoms of the reference molecule and the axis defined by the hydrogen atoms of the other molecule.

Note that, strictly due to geometrical considerations, an isotropic distribution of molecules around the reference molecule will yield a higher probability of angle $\theta_{\mathrm{CM}}$ being perpendicular rather than parallel. The same happens with angles $\alpha, \beta$, and $\gamma$, although it does not happen with $\varphi_{\mathrm{CM}}$. To prevent misleading figures arising from this fact, probability distributions have been plotted as a function of the angle or the cosine of the angle to ensure they will appear as flat functions for isotropic distributions.

\section{RESULTS AND DISCUSSION}

A first step for the analysis of the relative positions of the molecules in a liquid is to find out how many molecules can be found within the first neighbor shell. Figure 3 shows the pair-correlation function of the centers of mass of the TDCE molecules calculated from the simulations for the lowand high-density liquid, which is proportional to the probability of finding a molecule at a certain distance of a reference molecule. The first neighbor shell is defined as the molecules comprised within the first peak of the pair-correlation function. Figure 3 also shows the molecular coordination number for the two liquids, calculated from the integration of the pair-correlation function. Usually, since density decreases as temperature increases within the same phase, the $g_{\mathrm{CM}}$ minimum that defines the first neighbor shell moves to higher values of $r$ because the mean distance between the molecules

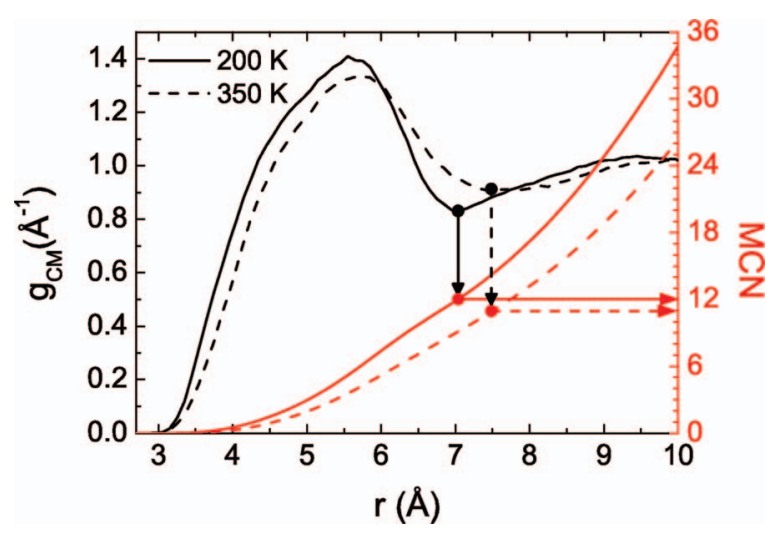

FIG. 3. Pair-correlation function of the centers of mass of the molecules ( $g_{\mathrm{CM}}$, black lines, scale on the left) and molecular coordination number (MCN, red lines, scale on the right) for the low- and high-density liquids (dashed and continuous lines, respectively) calculated from the simulation. The first neighbor shell contains 11 molecules in the case of the low-density liquid and 12 in the case of the high-density liquid.

is also increased. However, since the growth of the molecular coordination number with respect to the distance is also slower due to a lower density, the corresponding number of molecules in the first neighbor shell ends up being the same. In the present case, contrary to what would be commonly expected, a different number of molecules was found within the first neighbor shell for each liquid, 12 molecules in the case of the high-density liquid compared with only 11 in the case of the low-density one, an indication that the liquid is undergoing a change in the molecular short-range order and not only a density variation.

Another representation that can provide further insight into the difference between the first neighbor shells of the two liquids, because it takes into account the volume change of the spherical shell as the distance increases, is the density within the first neighbor shell as a function of the molecular coordination number (see Fig. 4). Using the molecular coordination number instead of $r$ as abscissa removes the expansion effects of increasing the temperature. In the high-density TDCE

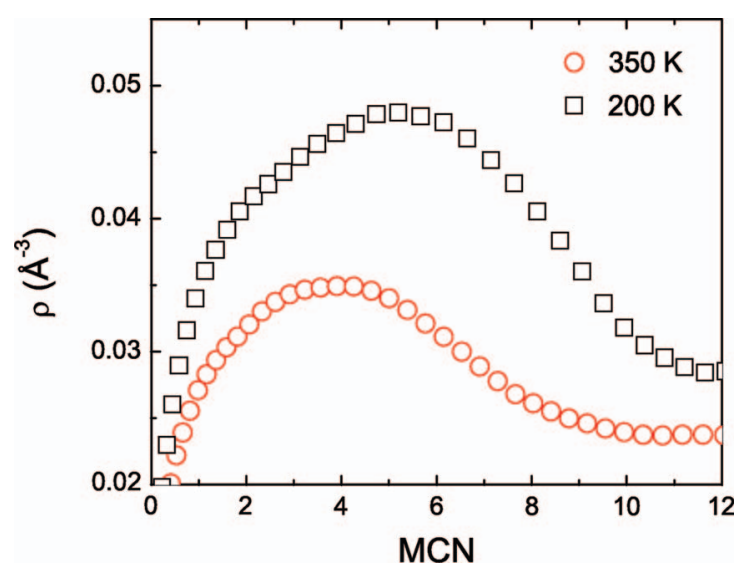

FIG. 4. Density within the first neighbor shell for the high- and low-density TDCE liquids (black squares and red circles, respectively) as a function of the molecular coordination number. A maximum is observed at the fifth and fourth neighbor for the high- and low-density TDCE liquids, respectively, which shows that there is a gap of one molecule in the structure patterns between both liquids and that the difference originates in the closest neighbors. 

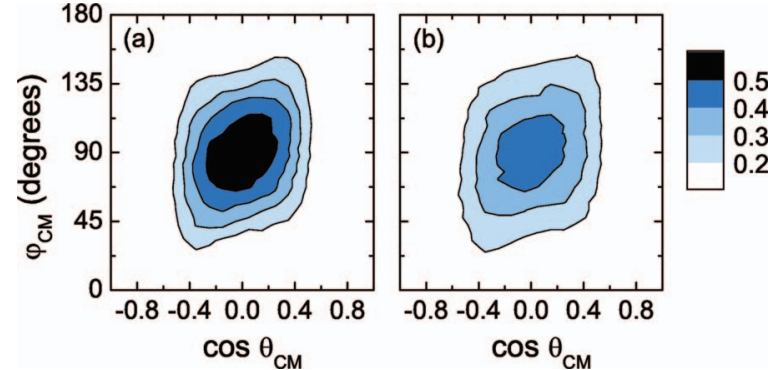

FIG. 5. Probability of the angular position $\left(\theta_{\mathrm{CM}}\right.$ and $\left.\varphi_{\mathrm{CM}}\right)$ of the center of mass of the first neighbor for the (a) high- and (b) low-density liquid (darker shade means higher probability). As expected, the high-density liquid is more structured, but there are no qualitative differences in the angular position of the first neighbor between both liquids (around $\theta_{\mathrm{CM}} \approx 90^{\circ}$ and $\varphi_{\mathrm{CM}} \approx 90^{\circ}$ ).

liquid the density increases up to the fifth neighbor and then starts to decrease, while in the case of the low-density TDCE liquid the same behavior is observed at the fourth neighbor. This is a good indication that the structural differences between both liquids are originating at the very first molecules.

A detailed analysis of the angular position of the centers of mass and relative orientation with respect to the reference molecule was performed for all molecules in the first neighbor shell. A strong agreement was found with the observation that there is a gap of one molecule in the structure patterns followed by both liquids, i.e., except for the first neighbor, the position and orientation of neighbor molecules in both liquids is qualitatively the same if every molecule in the low-density liquid is compared to the next molecule in the high-density liquid instead of comparing the same molecule in both liquids. The interested reader can turn to the supplementary material available online for more details on this result. ${ }^{23}$

Because the changes happen on the very first neighbors and the orientational correlations in molecular liquids are lost as distance increases, in this work we have focused on the analysis of just the single first neighbor, to which the following molecules will have to accommodate. ${ }^{24}$ Figure 5 shows the angular position $\left(\theta_{\mathrm{CM}}\right.$ and $\left.\varphi_{\mathrm{CM}}\right)$ of the center of mass of the first neighbor with respect to the reference molecule (for more details and examples on bivariate analysis see Refs. 25-28), where no qualitative difference has been observed between both liquids, since their first neighbor is equally located around $\theta_{\mathrm{CM}} \approx 90^{\circ}$ and $\varphi_{\mathrm{CM}} \approx 90^{\circ}$. Figure 6
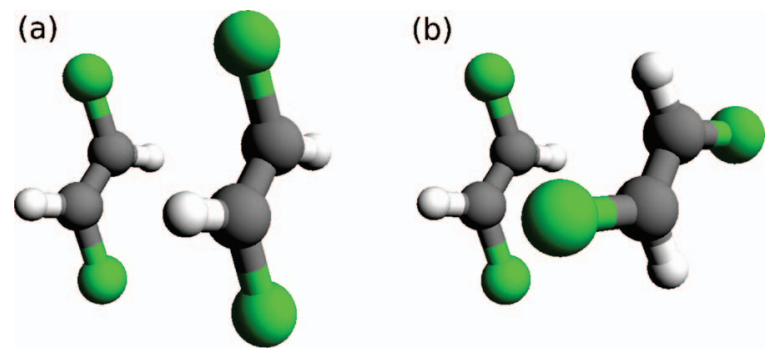

FIG. 6. Scheme of the two most likely configurations of the first neighbor and the reference molecule: (a) parallel or P, and (b) crossed or C. To illustrate the $\mathrm{P}$ configuration a molecular arrangement with $\alpha=0^{\circ}, \beta=0^{\circ}$, and $\gamma$ $=76.9^{\circ}$ has been chosen, while in the case of the $\mathrm{C}$ configuration it has been chosen with $\alpha=76.9^{\circ}, \beta=76.9^{\circ}$, and $\gamma=0^{\circ}$. Both examples have the same relative angular position $\left(\theta_{\mathrm{CM}}=90^{\circ}\right.$ and $\left.\varphi_{\mathrm{CM}}=90^{\circ}\right)$.

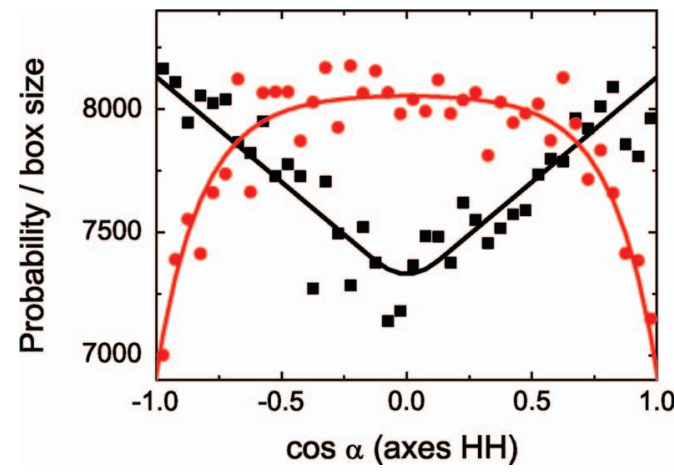

FIG. 7. Probability of the cosine of angle $\alpha$ of the first neighbor for the lowand high-density liquid (red dots and black squares, respectively). Lines are a guide to the eye. There is a difference in the relative orientation of the first neighbor with respect to the reference molecule between both liquids.

shows two examples of molecular pairs in such angular position. Although the high-density liquid is much more structured, it was a feature already expected due to the smaller effects of thermal agitation, and cannot be viewed as a meaningful difference in the structure.

Concerning the analysis of the relative orientation of the first neighbor with respect to the reference molecule, the probability distribution of the angle $\alpha$ (angle between the hydrogen axes of both molecules) of the first neighbor is shown in Fig. 7 for both liquids, where a discrepancy in the shape of the high- and low-density liquid distributions evidences an orientational contribution of the first neighbor to the differences in the molecular short-range order. No qualitative differences were observed when the same analyses were performed for the orientational angles $\beta$ and $\gamma$.

Although the observed angular position of the center of mass of the first neighbor with respect to the reference molecule between both densities is almost the same, the relative orientation between the molecules is conspicuously divergent, pointing out that this is where the key for the explanation must be sought.

Figure 7 shows that there is a discrepancy in the molecular orientation of the two liquids but it does not explain the origin of the density anomaly. ${ }^{10,11}$ Thus, a detailed analysis of the molecular orientations must be undertaken to understand the reasons behind the different molecular arrangements.

To learn which are the preferred relative orientations of the first neighbor and the reference molecule, a mapping of the configuration space is depicted in Fig. 8. This figure shows the probability distribution of the orientation between the first neighbor and the reference molecule (angles $\beta$ and $\gamma$ ) for the high- and low-density TDCE liquids. As expected in a highly disordered system, there is a continuum of possible configurations, although not all of them are equally probable. The configuration space has been divided into 16 regions of possible configurations, corresponding to the grid drawn in this figure. In both liquids, the same four regions (labeled $\mathrm{P}$ and $\mathrm{C}$ in Fig. 8) have a higher probability than the rest but, for molecular symmetry reasons, they account only for two possible configurations of the reference molecule and its first neighbor. The definition of each configuration includes all the molecular 

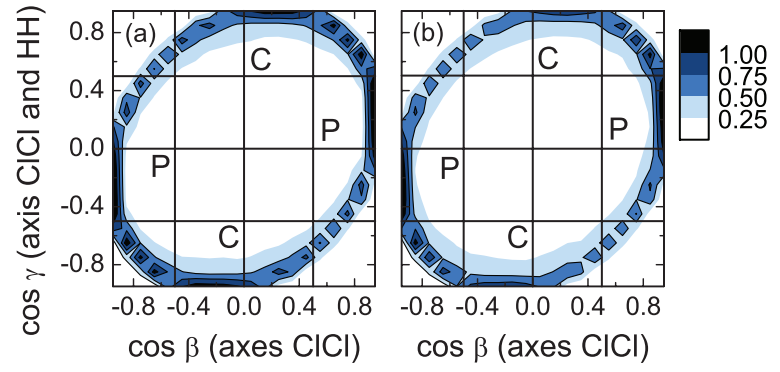

FIG. 8. Probability of combinations of $\beta$ and $\gamma$ cosines of the first neighbor for the (a) high- and (b) low-density liquids (darker shade means higher probability). The continuum of configurations between the first neighbor and the reference molecule have been grouped into 16 regions of possible configurations (grid), from which those labeled $\mathrm{P}$ and $\mathrm{C}$ are the most likely at both temperatures.

arrangements between the first neighbor and the reference molecule that contribute to the two equivalent regions in Fig. 8. The combinations of ranges of $\cos \beta$ and $\cos \gamma$ values that define the regions of $\mathrm{P}$ and $\mathrm{C}$ configurations are shown in Table I.

In Fig. 6, representative arrangements of molecular pairs in the $\mathrm{P}$ and $\mathrm{C}$ configurations have been depicted to provide a scheme of the relative orientation between the first neighbor and the reference molecule. As can be seen in this figure, molecular pairs with parallel chlorine axes and parallel hydrogen axes are denoted as P configuration (Fig. 6(a)), while molecules with their chlorine axis parallel to the hydrogen axis of the reference molecule and vice versa (crossed molecules) are denoted as $\mathrm{C}$ configuration (Fig. 6(b)).

Although qualitatively Figs. 8(a) and 8(b) appear to be very similar, a quantitative analysis shows that for the lowdensity liquid, configuration $P$ represents a higher fraction of first neighbors than configuration $\mathrm{C}$. This situation is reversed in the high-density liquid (see Table II). Hence, as the temperature is changed, there is an inversion of the dominant population in the liquid.

Could this difference of population in the configurations of the first neighbor between the low- and high-density liquid be the underlying cause of the observed anomalies between both densities? To ascertain whether having the first neighbor in different configurations can explain the observed shortrange order discrepancies of liquid TDCE (and its dynamic properties), a separate analysis has been performed to each configuration to compare their particularities.

As it has been previously explained, Fig. 5 shows the most probable relative position of the molecular centers of mass when the first neighbors in any of the configurations are taken into account. To obtain more detailed information

TABLE I. Range of values used to define which molecular pairs are in $\mathrm{P}$ or C configuration.

\begin{tabular}{lcc}
\hline \hline Configuration & $\cos \beta$ & $\cos \gamma$ \\
\hline $\mathrm{P}$ & 0.5 to 1.0 & 0.0 to 0.5 \\
$\mathrm{P}$ & -1.0 to -0.5 & -0.5 to 0.0 \\
$\mathrm{C}$ & 0.0 to 0.5 & 0.5 to 1.0 \\
$\mathrm{C}$ & -0.5 to 0.0 & -1.0 to -0.5 \\
\hline \hline
\end{tabular}

TABLE II. Ratios of first neighbor molecules in $\mathrm{P}$ and $\mathrm{C}$ configurations with respect to the total number of first neighbor molecules. $\Delta$ represents the increment of the fraction of molecules in that configuration with respect to the total number of molecules, as the temperature is decreased from 350 to $200 \mathrm{~K}$.

\begin{tabular}{lccc}
\hline \hline Configuration & $350 \mathrm{~K}$ & $200 \mathrm{~K}$ & $\Delta$ \\
\hline $\mathrm{P}$ & 0.19002 & 0.17494 & $-1.5 \%$ \\
$\mathrm{C}$ & 0.17648 & 0.19880 & $+2.2 \%$ \\
\hline \hline
\end{tabular}

from this magnitude, in Fig. 9 the probability density functions of the same angles $\left(\theta_{\mathrm{CM}}\right.$ and $\left.\varphi_{\mathrm{CM}}\right)$ have been evaluated but only for first neighbors in configurations $\mathrm{P}$ and $\mathrm{C}$, making a distinction between both configurations. From Fig. 9, it is inferred that first neighbors in $\mathrm{P}$ and $\mathrm{C}$ configuration tend to locate themselves roughly around $\theta_{\mathrm{CM}} \approx 90^{\circ}$ and $\varphi_{\mathrm{CM}} \approx 90^{\circ}$ regardless of their configuration (the same position seen in the analysis of all first neighbors in Fig. 5). Further analyses of the remaining configurations showed that this is the preferred arrangement of all first neighbors and not only of those in configurations $\mathrm{P}$ and $\mathrm{C}$.

Nevertheless, the angular position distributions of the first neighbors are not completely equivalent for both configurations. In the high-density liquid (Figs. 9(a) and 9(b)), where the difference between configurations is more easily observed, $\theta_{\mathrm{CM}}$ and $\varphi_{\mathrm{CM}}$ distributions for the $\mathrm{C}$ configuration peak clearly at $90^{\circ}$ while the $\mathrm{P}$ configuration displays a double peak for the $\theta_{\mathrm{CM}}$ and a flat top for the $\varphi_{\mathrm{CM}}$ distribution that spans to a wider range of values. This means that molecular pairs in $\mathrm{C}$ configuration sit comfortably in front of each

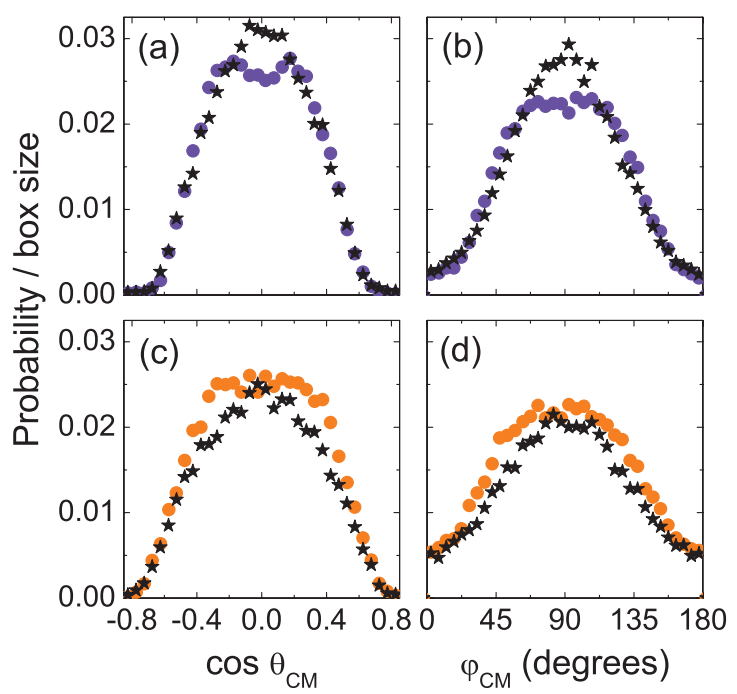

FIG. 9. Contribution of configurations $\mathrm{P}$ (circles) and $\mathrm{C}$ (stars) to the probability of the center of mass position of the first neighbor molecule with respect to the reference molecule. (a) and (b) show the cosine of $\theta_{\mathrm{CM}}$ and $\varphi_{\mathrm{CM}}$, respectively, for the high-density liquid. And (c) and (d) show the cosine of $\theta_{\mathrm{CM}}$ and $\varphi_{\mathrm{CM}}$, respectively, for the low-density liquid. The center of mass of the molecules in the $\mathrm{C}$ configuration tends to be exactly in front of the reference molecule $\left(\theta_{\mathrm{CM}}\right.$ and $\left.\varphi_{\mathrm{CM}}=90^{\circ}\right)$ while the center of mass of the molecules in the $\mathrm{P}$ configuration tends to be slightly on a side or is evenly distributed on a higher range of angles around this value. For the high-density liquid, the total contribution of the $\mathrm{C}$ configuration (area in this graph) is greater than the contribution from the $\mathrm{P}$ configuration. This situation is reversed in the low-density liquid. 


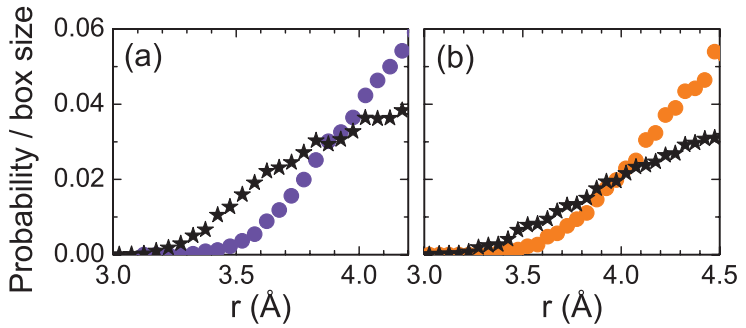

FIG. 10. Contribution of configurations $\mathrm{P}$ (circles) and $\mathrm{C}$ (stars) to the probability of the center of mass distance of the first neighbor molecule with respect to the reference molecule for the (a) high- and (b) low-density liquid. Irrespective of temperature, the centers of mass of molecules in the $\mathrm{C}$ configuration are on average closer to the reference molecule than in the $\mathrm{P}$ configuration.

other while molecules in configuration P prefer to be slightly displaced from that central position.

Probability distribution functions in Fig. 9 have been normalized to the total number of molecules so that their area is directly proportional to the number of molecules in that configuration, to make the contribution to the total probability of each configuration clear. Consequently, in this figure it is easier than in Fig. 8 to appreciate, from the area of the distributions, that in the high-density liquid the contribution of $\mathrm{C}$ configuration is higher than the $\mathrm{P}$ configuration, and that in the low-density liquid it is lower.

In addition to the difference in angular position between molecules in configurations $\mathrm{C}$ and $\mathrm{P}$, the distance between first neighbors in one or the other configuration also varies significantly. Figure 10 shows the contribution to the probability that a first neighbor is at a certain distance from a reference molecule for each of the configurations for the high- and low-density liquids. As can be seen in this figure, the centers of mass of molecules in the $\mathrm{C}$ configuration are on average closer than in the $\mathrm{P}$ configuration, showing that molecules in $\mathrm{C}$ configuration are more tightly bonded than those in $\mathrm{P}$ configuration.

Abundance of a configuration with a stronger bond between molecules means that on average they are closer to each other, and a more ordered arrangement of the molecules makes it even easier to pack them tighter and to fit in the first neighbor shell the additional molecule that we have observed in the high-density liquid.

The fact that there is a favored local structure is in agreement with the previous works. ${ }^{9-12,15}$

\section{CONCLUSIONS}

We have unraveled the structural causes behind the density and spin-relaxation time singularities, and the viscosity and slope of the rotational relaxation time discontinuities in the TDCE liquid by means of a detailed analysis of the molecular short-range order.

Except for the first neighbor, the position and orientation of neighbor molecules in both liquids have been found to be qualitatively the same if an offset of one molecule is taken into account. The first neighbor shell in the low-density TDCE liquid contains 11 molecules. An additional molecule is contained in the first neighbor shell of the high-density liquid, adding up to 12 molecules. According to the density distribution analysis, the difference in the molecular arrangement must originate in the closest four to five molecules of the first neighbor shell.

The angular position of the center of mass of the first neighbor is almost identical for both liquids, molecules gather around $\theta_{\mathrm{CM}} \approx 90^{\circ}$ and $\varphi_{\mathrm{CM}} \approx 90^{\circ}$. But a strong difference is observed in the distribution of $\alpha$, the angle between the hydrogen axes, regarding the relative orientation of the molecular pairs.

Further analysis of the relative molecular orientation of the first neighbor and the reference molecule reveals that two of the configurations that have been defined are more probable than the rest (parallel, $\mathrm{P}$, and crossed, C). While the $\mathrm{P}$ configuration prevails in the low-density liquid, the $\mathrm{C}$ configuration prevails in the high-density one. There is an inversion of the configuration populations when the low-density TDCE liquid turns into the high-density one and vice versa.

The first neighbors in $\mathrm{C}$ configuration have a tendency to locate themselves closer to the reference molecule and exactly at the angular position $\theta_{\mathrm{CM}}=90^{\circ}$ and $\varphi_{\mathrm{CM}}=90^{\circ}$ displaying strong bonding. In contrast, the first neighbors in $\mathrm{P}$ configuration tend to be further apart and with their centers of mass slightly displaced from that angular position.

Hydrogen bonding between the chlorine atoms of one molecule and the hydrogen atoms of the other explains why molecular pairs in C configuration can bond more tightly than those in $\mathrm{P}$ configuration.

A larger proportion of first neighbors in $\mathrm{C}$ configuration, that can sit exactly in front of the reference molecule in an orderly manner and much closer, facilitates a better packing, so that an additional molecule fits in the first coordination shell of the high-density TDCE liquid, as it has been observed. This can also explain the density anomaly between the high- and low-density TDCE liquids.

More structured molecular pairs given by a stronger bonding of the first neighbors in $\mathrm{C}$ configuration is likely to be also the key to the explanation of the dynamical anomalies reported in previous studies. ${ }^{9-12,15}$

\section{ACKNOWLEDGMENTS}

The authors would like to thank X. Ariza for the deuteration of the sample. This work was supported by the Spanish Ministry of Science and Innovation (FIS2008-00837) and by the Government of Catalonia (2009SGR-1251).

${ }^{1}$ C. Talón, M. A. Ramos, S. Vieira, G. J. Cuello, F. J. Bermejo, A. Criado, M. L. Senent, S. M. Bennington, H. E. Fischer, and H. Schober, Phys. Rev. B 58, 745 (1998).

${ }^{2}$ P. S. Salmon, R. A. Martin, P. E. Mason, and G. J. Cuello, Nature (London) 435, 75 (2005)

${ }^{3}$ O. Gereben, P. Jovari, L. Temleitner, and L. Pusztai, in Proceedings of 3 rd International Conference on Amorphous and Nanostructured Chalcogenides, Brasov, Romania, July 02-06, 2007 [J. Optoelectron, Adv. Mater. 9, 3021 (2007)], online at http://joam.inoe.ro/download.php?idu=953.

${ }^{4}$ S. Pothoczki, L. Temleitner, and L. Pusztai, J. Chem. Phys. 134, 044521 (2011).

${ }^{5}$ A. K. Soper, Chem. Phys. 202, 295 (1996).

${ }^{6}$ A. K. Soper, Chem. Phys. 258, 121 (2000).

${ }^{7}$ M. P. Allen and D. J. Tildesley, Computer Simulation of Liquids (Clarendon, New York, NY, 1989). 
${ }^{8}$ M. Haughney, M. Ferrario, and I. R. McDonald, J. Phys. Chem. 91, 4934 (1987).

${ }^{9}$ S. Kawanishi, T. Sasuga, and M. Takehisa, J. Phys. Soc. Jpn. 50, 3080 (1981).

${ }^{10}$ S. Kawanishi, T. Sasuga, and M. Takehisa, J. Phys. Soc. Jpn. 51, 1579 (1982).

${ }^{11}$ B. Zegrodnik, A. Hacura, W. Carius, and O. Schröter, Acta Phys. Pol. 75, 469 (1989).

${ }^{12}$ K. Merkel, A. Kocot, R. Wrzalik, and J. Ziolo, J. Chem. Phys. 129, 074503 (2008).

${ }^{13}$ D. A. Turton, D. F. Martin, and K. Wynne, Phys. Chem. Chem. Phys. 12, $4191(2010)$

${ }^{14}$ S. Rzoska, J. Ziolo, A. Drozd-Rzoska, J. Ll. Tamarit, and N. Veglio, in Proceedings of the European-Science-Foundation Exploratory Workshop on Glassy Liquids Under Pressure, Ustron, Poland, October 10-12, 2007 [J. Phys.: Condens. Matter 20, 244124 (2008)].

${ }^{15}$ M. Rovira-Esteva, A. Murugan, L. C. Pardo, S. Busch, M. D. RuizMartin, M. S. Appavou, J. Ll. Tamarit, C. Smuda, T. Unruh, F. J. Bermejo, G. J. Cuello, and S. J. Rzoska, Phys. Rev. B 81, 092202 (2010).

${ }^{16}$ H. E. Fischer, G. J. Cuello, P. Palleau, D. Feltin, A. C. Barnes, Y. S. Badyal, and J. M. Simonson, in Proceedings of the International Conference on Neutron Scattering, Munich, Germany, Sep 09-13, 2001 [Appl. Phys. A: Mater. Sci. Process. 74, S160 (2002)].

${ }^{17}$ T. C. Hansen, P. F. Henry, H. E. Fischer, J. Torregrossa, and P. Convert, Meas. Sci. Technol. 19, 034001 (2008).

${ }^{18} \mathrm{M}$. Howe, R. McGreevy, and P. Zetterström, Computer code CORRECT, correction program for neutron diffraction data, Internal Report, NFL Studsvik, Uppsala, Sweden, 1996.
${ }^{19}$ J. Wang, R. Wolf, J. Caldwell, P. Kollman, and D. Case, J. Comput. Chem. 25, 1157 (2004)

${ }^{20}$ D. A. Case, T. E. CheathamIII, C. L. Simmerling, J. Wang, R. E. Duke, R. Luo, K. M. Merz, B. Wang, D. A. Pearlman, M. Crowley, S. Brozell, V. Tsui, H. Gohlke, J. Mongan, V. Hornak, G. Cui, P. Beroza, C. Schafmeister, J. W. Caldwell, W. S. Ross, and P. A. Kollman, AMBER 8, University of California, San Francisco, 2004.

${ }^{21}$ N. A. Murugan and H. W. Hugosson, J. Phys. Chem. B 113, 1012 (2009).

${ }^{22}$ N. A. Murugan and H. W. Hugosson, Phys. Chem. Chem. Phys. 10, 6135 (2008).

${ }^{23}$ See supplementary material at http://dx.doi.org/10.1063/1.3697849 for full details on the observation that there is a gap of one molecule in the structure patterns followed by both liquids.

${ }^{24}$ L. C. Pardo, J. Ll. Tamarit, N. Veglio, F. J. Bermejo, and G. J. Cuello, Phys. Rev. B 76, 134203 (2007).

${ }^{25}$ M. Rovira-Esteva, L. C. Pardo, J. Ll. Tamarit, and F. J. Bermejo, in Metastable Systems Under Pressure, NATO Science for Peace and Security Series A: Chemistry and Biology, edited by S. J. Rzoska, A. Drozd-Rzoska, and V. Mazur (Springer, Netherlands, 2009), pp. 63-77.

${ }^{26}$ L. C. Pardo, M. Rovira-Esteva, J. Ll. Tamarit, N. Veglio, F. J. Bermejo, and G. J. Cuello, in Metastable Systems Under Pressure, NATO Science for Peace and Security Series A: Chemistry and Biology, edited by S. J. Rzoska, A. Drozd-Rzoska, and V. Mazur (Springer, Netherlands, 2009), pp. 79-91.

${ }^{27}$ M. Rovira-Esteva, N. A. Murugan, L. C. Pardo, S. Busch, J. Ll. Tamarit, Sz. Pothoczki, G. J. Cuello, and F. J. Bermejo, Phys. Rev. B 84, 064202 (2011)

${ }^{28}$ Sz. Pothoczki, A. Ottochian, M. Rovira-Esteva, L. C. Pardo, J. Ll. Tamarit, and G. J. Cuello, Phys. Rev. B 85, 014202 (2012). 